\title{
Erratum to: A Multicenter, Randomized Clinical Trial to Assess the Efficacy of a Therapeutic Intervention Program for Caregivers of People with Dementia
}

Rayoung Yoo ${ }^{a}$, Jiyoung Yeom ${ }^{a}$, Geon Ha Kimª, Hee Kyung Parkª ${ }^{a}$ Yeonwook Kang ${ }^{\mathrm{b}, \mathrm{c}}$, Jihye Hwang ${ }^{\mathrm{d}}$, Seong Hye Choi ${ }^{\mathrm{e}}$, Hae Ri Na ${ }^{f}$, Soo-Jin $\mathrm{Cho}^{g}$, Kyung-Ho $\mathrm{Yu}^{\mathrm{c}}$, Do Hoon Kim ${ }^{\mathrm{h}}$, Jae-Hong Lee', Jee Hyang Jeong ${ }^{\mathrm{a}}$

\author{
aDepartment of Neurology, Ewha Womans University Mokdong Hospital, Ewha Womans University School of Medicine, Seoul, Korea \\ ${ }^{b}$ Department of Psychology, Hallym University, Chuncheon, Korea \\ 'Department of Neurology, Hallym University Sacred Heart Hospital, Anyang, Korea \\ dDepartment of Neurology, Keimyung University College of Medicine, Dongsan Medical Center, Daegu, Korea \\ ${ }^{e}$ Department of Neurology, Inha University School of Medicine, Incheon, Korea \\ 'Department of Neurology, Bobath Memorial Hospital, Seongnam, Korea \\ ${ }^{9}$ Department of Neurology, Hallym University Dongtan Sacred Heart Hospital, Hwaseong, Korea \\ hDepartment of Psychiatry, Hallym University Chuncheon Sacred Heart Hospital, Chuncheon, Korea \\ 'Department of Neurology, Asan Medical Center, University of Ulsan College of Medicine, Seoul, Korea
}

J Clin Neurol 2019;15(2):235-242 / https://doi.org/10.3988/jcn.2019.15.2.235

Unfortunately, the original version of this article had errors in the data displayed in flow diagram.

In the Fig. 1, The author wishes to delete $(n=2)$ and $(n=4)$ from the Withdrew Category as shown on the left.

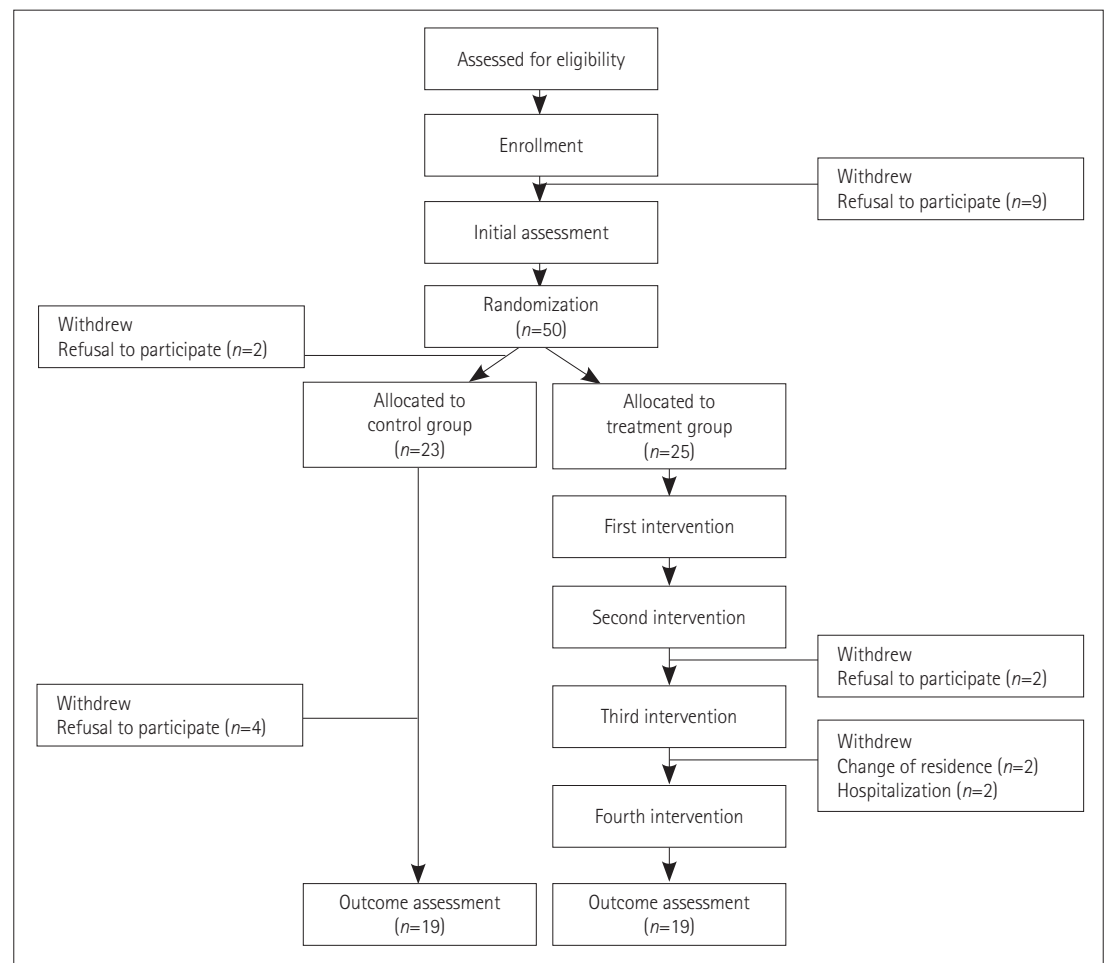

@ This is an Open Access article distributed under the terms of the Creative Commons Attribution Non-Commercial License (https://creativecommons.org/ licenses/by-nc/4.0) which permits unrestricted non-commercial use, distribution, and reproduction in any medium, provided the original work is properly cited. 